\title{
EMBEDDABILITY OF RIGHT-ANGLED ARTIN GROUPS ON THE COMPLEMENTS OF LINEAR FORESTS
}

\author{
TAKUYA KATAYAMA
}

\begin{abstract}
In this article, we prove that embeddings of right-angled Artin group $A_{1}$ on the complement of a linear forest into another right-angled Artin group $A_{2}$ can be reduced to full embeddings of the defining graph of $A_{1}$ into the extension graph of the defining graph of $A_{2}$.
\end{abstract}

\section{INTRODUCTION}

Let $\Gamma$ be a simple graph (abbreviated a graph). We denote the vertex set and the edge set of $\Gamma$ by $V(\Gamma)$ and $E(\Gamma)$, respectively. The right-angled Artin group on $\Gamma$ is the group given by the following presentation:

$$
\left.A(\Gamma)=\langle V(\Gamma)| v_{i} v_{j} v_{i}^{-1} v_{j}^{-1}=1 \text { if }\left\{v_{i}, v_{j}\right\} \in E(\Gamma)\right\rangle .
$$

A graph homomorphism is a map between the vertex sets of two graphs, which maps adjacent vertices to adjacent vertices. An injective graph homomorphism (abbreviated an embedding) $\iota: \Lambda \rightarrow \Gamma$ is called full if $\iota$ maps non-adjacent vertices to non-adjacent vertices. If there is a full embedding $\iota: \Lambda \rightarrow \Gamma$, then we denote by $\Lambda \leq \Gamma$ and $\Lambda$ is called a full subgraph of $\Gamma$. For finite graphs $\Lambda$ and $\Gamma$, it is wellknown that $A(\Lambda)$ is isomorphic to $A(\Gamma)$ as a group if and only if $\Lambda$ is isomorphic to $\Gamma$ as a graph. Following S. Kim and T. Koberda 4, the extension graph $\Gamma^{e}$ of a finite graph $\Gamma$ is the graph such that the vertex set of $\Gamma^{e}$ consists of the words in $A(\Gamma)$ that are conjugate to the vertices of $\Gamma$, and two vertices of $\Gamma^{e}$ are joined by an edge if and only if they are commutative as words in $A(\Gamma)$. A celebrated theorem due to Kim-Koberda states that, if a finite graph $\Lambda$ is a full subgraph of the extension graph $\Gamma^{e}$ of a finite graph $\Gamma$, then we have an injective homomorphism (abbreviated an embedding) $A(\Lambda) \hookrightarrow A(\Gamma)$. In this article, a path graph $P_{n}$ on $n(\geq 1)$ vertices is the graph whose underlying space is homeomorphic to the origin $\{0\}$ or unit interval $[0,1]$ in the 1-dimensional Euclidean space. A linear forest is the disjoint union of finitely many path graphs. The complement $\Lambda^{c}$ of a graph $\Lambda$ is the graph consisting of the vertex set $V\left(\Lambda^{c}\right)=V(\Lambda)$ and the edge set $E\left(\Lambda^{c}\right)=\{\{u, v\} \mid u, v \in V(\Lambda),\{u, v\} \notin E(\Lambda)\}$.

In [3] the author "proved" the following theorem.

Theorem 1.1. Let $\Lambda$ be the complement of a linear forest and $\Gamma$ a finite graph. If $A(\Lambda) \hookrightarrow A(\Gamma)$, then $\Lambda \leq \Gamma$.

We remark that Theorem 1.1 is equivalent to [3, Theorem 1.3(1)]. In the proof of [3, Theorem 1.3(1)], the author used the following "Theorem" (see the second line of the proof of Theorem 3.6 in [3]).

Date: May 16, 2022.

2010 Mathematics Subject Classification. Primary: 20F36.

Key words and phrases. Right-angled Artin group. 
Theorem 1.2 ([1, Theorem 3.14]). Let $\Lambda$ be the complement of a forest and $\Gamma$ a finite graph. If $A(\Lambda) \hookrightarrow A(\Gamma)$, then $\Lambda \leq \Gamma^{e}$.

However, E. Lee and S. Lee 5 pointed out that the above "Theorem" is incorrect by giving a counter-example. Thus the author's proof of Theorem 1.1 in 3 is not valid.

The purpose of this article is to give a complete proof of Theorem 1.1 by establishing the following theorem which shows that "Theorem 1.2' holds when $\Lambda$ is the complement of a linear forest.

Theorem 1.3. Let $\Lambda$ be the complement of a linear forest and $\Gamma$ a finite graph. If $A(\Lambda) \hookrightarrow A(\Gamma)$, then $\Lambda \leq \Gamma^{e}$.

In fact, the author applied "Theorem [1.2' only for the complement of linear forests in the proof of Theorem 1.3(1) in [3].

We note that this theorem gives a partial positive answer to the following question.

Question 1.4 ([4, Question 1.5]). For which graphs $\Lambda$ and $\Gamma$ do we have $A(\Lambda) \hookrightarrow$ $A(\Gamma)$ only if $\Lambda \leq \Gamma^{e}$ ?

With regard to this question, the reader is referred to the introduction of the paper [5, Question 1.5] due to Lee-Lee.

This article is organized as follows. In Section 2, we introduce terminology and known results. For the sake of convenience, we discuss relation between graph-join (a certain graph operation) and embedding problems in Section 3 Section 4 is devoted to the proof of Theorem 1.3

Acknowledgements. The author would like to thank his supervisor, Makoto Sakuma, for helpful discussions and a number of improvements regarding this paper. The author thanks to Eon-kyung Lee and Sang-jin Lee for their encouragements.

\section{Preliminaries}

Suppose that $\Gamma$ is a graph. An element of $V(\Gamma) \cup V(\Gamma)^{-1}$ is called a letter. Any element in $A(\Gamma)$ can be expressed as a word, which is a finite multiplication of letters. Let $w=a_{1} \cdots a_{l}$ be a word in $A(\Gamma)$ where $a_{1}, \ldots, a_{l}$ are letters. We say $w$ is reduced if any other word representing the same element as $w$ in $A(\Gamma)$ has at least $l$ letters. The following lemma is useful for checking whether a given word is reduced or not (cf. [2, Section 5]).

Lemma 2.1. Let $w$ be a word in $A(\Gamma)$. Then $w$ is reduced if and only if $w$ does not contain a word of the form $v^{\epsilon} x v^{-\epsilon}$, where $v$ is a vertex of $\Gamma, \epsilon= \pm 1$ and $x$ is a word such that $v$ is commutative with all of the letters in $x$.

The support of a reduced word $w$ is the smallest subset $S$ of $V(\Gamma)$ such that each letter of $w$ is in $S$ or $S^{-1}$; we write $S=\operatorname{supp}(w)$. It is well-known that the support does not depend on the choice of a reduced word, and so we can define the support of an element of $A(\Gamma)$. By a clique, we mean a complete subgraph of a graph. We rephrase a special case of Servatius' Centralizer Theorem [6, The Centralizer Theorem in Section III] as follows.

Lemma 2.2. Let $w_{1}$ and $w_{2}$ be reduced words in $A(\Gamma)$ whose supports span cliques in $\Gamma$. Then the words $w_{1}$ and $w_{2}$ are commutative if and only if $\operatorname{supp}\left(w_{1}\right)$ and $\operatorname{supp}\left(w_{2}\right)$ are contained in a single clique in $\Gamma$. 
In this article, we say that a homomorphism $\psi: A(\Lambda) \rightarrow A(\Gamma)$ between two rightangled Artin groups satisfies $(K K)$ condition if $\operatorname{supp}(\psi(v))$ consists of mutually adjacent vertices in $\Gamma$ for all $v \in V(\Lambda)$ (i.e., $\operatorname{supp}(\psi(v))$ spans a clique in $\Gamma$ ).

Theorem 2.3 (4, Theorem 4.3]). Suppose that $\Lambda$ and $\Gamma$ are finite graphs and $A(\Lambda) \hookrightarrow A(\Gamma)$. Then there is an embedding $\psi: A(\Lambda) \hookrightarrow A\left(\Gamma^{e}\right)$ such that $\psi$ satisfies $(K K)$ condition. Namely, for all $v \in V(\Lambda), \operatorname{supp}(\psi(v))$ consists of mutually adjacent vertices in $\Gamma^{e}$.

\section{GRAPH-JOIN}

In this section, we prove Proposition 3.2, which says that, for two finite graphs $\Lambda$ and $\Gamma$ such that there is an embedding $A(\Lambda) \hookrightarrow A(\Gamma)$ satisfying condition (KK), the problem of finding a full embedding $\Lambda \rightarrow \Gamma$, with a certain restriction, is reduced to the corresponding problems for the "join-components" of $\Lambda$. The (graph-)join $\Lambda_{1} * \cdots * \Lambda_{m}$ of graphs $\Lambda_{1}, \ldots, \Lambda_{m}$ is the graph obtained from the disjoint union $\Lambda_{1} \sqcup \cdots \sqcup \Lambda_{m}$ by joining the vertices $u$ and $v$ for all $u \in V\left(\Lambda_{i}\right), v \in V\left(\Lambda_{j}\right)(i \neq j)$. In this article, we say that a graph $\Lambda$ is irreducible (with respect to join) if $\Lambda$ cannot be the join of two non-empty graphs. Any finite graph $\Lambda$ is the join of finitely many irreducible graphs. Indeed, this follows from the fact that $\Lambda=\Lambda_{1} * \cdots * \Lambda_{m}$ if and only if $\Lambda^{c}=\Lambda_{1}^{c} \sqcup \cdots \sqcup \Lambda_{m}^{c}$. This fact also implies the following lemma.

Lemma 3.1. A finite graph $\Lambda$ is irreducible if and only if $\Lambda^{c}$ is connected. In particular, if $\Lambda$ is an irreducible graph containing at least two vertices, then for any vertex $u \in V(\Lambda)$, there is a vertex $u^{\prime} \in V(\Lambda)$ such that $u$ and $u^{\prime}$ are non-adjacent.

Proof. Suppose that $\Lambda$ is an irreducible graph containing at least two vertices. Pick a vertex $u \in V(\Lambda)$. If $u$ does not have a non-adjacent vertex, then we have a decomposition $\Lambda=\{u\} * \check{\Lambda}$, where $\check{\Lambda}$ is a full subgraph spanned by $V(\Lambda) \backslash\{u\}$, a contradiction.

Besides, the right-angled Artin group on the join, $A\left(\Lambda_{1} * \cdots * \Lambda_{m}\right)$, is isomorphic to the direct product $A\left(\Lambda_{1}\right) \times \cdots \times A\left(\Lambda_{m}\right)$. For simplicity, if $\psi: A(\Lambda) \rightarrow A(\Gamma)$ is a homomorphism, then by $\operatorname{supp}(\psi)$ we denote $\cup_{v \in V(\Lambda)} \operatorname{supp}(\psi(v))$.

Proposition 3.2. Let $\Lambda$ be the join $\Lambda_{1} * \cdots * \Lambda_{m}$ of finite irreducible graphs $\Lambda_{1}, \ldots, \Lambda_{m}$, and let $\Gamma$ be a finite graph. Suppose that the following conditions hold:

(1) There is an embedding $\psi: A(\Lambda) \hookrightarrow A(\Gamma)$ satisfying condition (KK).

(2) For each $1 \leq i \leq m$, there is a full embedding $\iota_{i}: \Lambda_{i} \rightarrow \Gamma$ with $\iota_{i}\left(\Lambda_{i}\right) \subset$ $\operatorname{supp}\left(\psi_{i}\right)$, where $\psi_{i}$ is the restriction of $\psi$ to $A\left(\Lambda_{i}\right)$.

Then there is a full embedding $\iota: \Lambda \rightarrow \Gamma$ with $\iota(\Lambda) \subset \operatorname{supp}(\psi)$.

We first prove this proposition in a special case.

Lemma 3.3. Let $\Lambda_{1}$ be a finite irreducible graph containing at least two vertices, and let $\Lambda_{2}$ and $\Gamma$ be finite graphs. Suppose that the following conditions hold:

(1) There is an embedding $\psi: A\left(\Lambda_{1} * \Lambda_{2}\right) \hookrightarrow A(\Gamma)$ satisfying condition (KK).

(2) For $i=1,2$, there are full embeddings $\iota_{i}: \Lambda_{i} \rightarrow \Gamma$ with $\iota_{i}\left(\Lambda_{i}\right) \subset \operatorname{supp}\left(\psi_{i}\right)$, where $\psi_{i}$ is the restriction of $\psi$ to $A\left(\Lambda_{i}\right)$.

Then the map $\iota: \Lambda_{1} * \Lambda_{2} \rightarrow \Gamma$, defined by $\iota(v)=\iota_{1}(v)$ or $\iota_{2}(v)$ according to whether $v \in V\left(\Lambda_{1}\right)$ or $v \in V\left(\Lambda_{2}\right)$, is a full embedding with $\iota\left(\Lambda_{1} * \Lambda_{2}\right) \subset \operatorname{supp}(\psi)$. 
Proof. We have only to prove: (i) $\iota_{1}\left(\Lambda_{1}\right) \cap \iota_{2}\left(\Lambda_{2}\right)=\emptyset$ and (ii) $\forall u \in V\left(\Lambda_{1}\right), \forall v \in$ $V\left(\Lambda_{2}\right), \iota_{1}(u)$ and $\iota_{2}(v)$ are adjacent in $\Gamma$. In fact (i) and (ii) imply that the map $\iota: \Lambda_{1} * \Lambda_{2} \rightarrow \Gamma$ is a full embedding. Moreover, the assumptions $\iota_{i}\left(\Lambda_{i}\right) \subset \operatorname{supp}\left(\psi_{i}\right)$ imply that the full embedding $\iota$ satisfies the desired property that $\iota\left(\Lambda_{1} * \Lambda_{2}\right) \subset$ $\operatorname{supp}(\psi)$.

(i) Pick $u_{1} \in V\left(\Lambda_{1}\right)$ and $u_{2} \in V\left(\Lambda_{2}\right)$. Since $\Lambda_{1}$ is irreducible and has at least two vertices, $\Lambda_{1}$ has a vertex $u_{1}^{\prime}$ which is non-adjacent to $u_{1}$ in $\Lambda_{1}$ by Lemma 3.1. Since $u_{1}$ is not adjacent to $u_{1}^{\prime}$ in $\Lambda_{1}$, and since $\iota_{1}: \Lambda_{1} \rightarrow \Gamma$ is full, $\iota_{1}\left(u_{1}\right)$ is not adjacent to $\iota_{1}\left(u_{1}^{\prime}\right)$ in $\Gamma$. On the other hand, we can prove that $\iota_{2}\left(u_{2}\right)$ is either identical with $\iota_{1}\left(u_{1}^{\prime}\right)$ or adjacent to $\iota_{1}\left(u_{1}^{\prime}\right)$ in $\Gamma$ as follows (and so $\iota_{1}\left(u_{1}\right) \neq \iota_{2}\left(u_{2}\right)$ in any case). By the assumptions that $\iota_{i}\left(\Lambda_{i}\right) \subset \operatorname{supp}\left(\psi_{i}\right)(i=1,2)$, there are vertices $\bar{u}_{1}^{\prime} \in V\left(\Lambda_{1}\right)$ and $\bar{u}_{2} \in V\left(\Lambda_{2}\right)$ such that $\iota_{1}\left(u_{1}^{\prime}\right) \in \operatorname{supp}\left(\psi\left(\bar{u}_{1}^{\prime}\right)\right)$ and $\iota_{2}\left(u_{2}\right) \in \operatorname{supp}\left(\psi\left(\bar{u}_{2}\right)\right)$. Moreover, since $\Lambda_{1}$ and $\Lambda_{2}$ are joined in $\Lambda_{1} * \Lambda_{2}$, the image $\psi\left(\bar{u}_{2}\right)$ is commutative with $\psi\left(\bar{u}_{1}^{\prime}\right)$, and therefore $\operatorname{supp}\left(\psi\left(\bar{u}_{2}\right)\right)$ and $\operatorname{supp}\left(\psi\left(\bar{u}_{1}^{\prime}\right)\right)$ are contained in a single clique by (KK) condition and Lemma 2.2. Thus $\iota_{2}\left(u_{2}\right)$ is either adjacent to $\iota_{1}\left(u_{1}^{\prime}\right)$ or identical with $\iota_{1}\left(u_{1}^{\prime}\right)$ in $\Gamma$.

(ii) Pick $u_{1} \in V\left(\Lambda_{1}\right)$ and $u_{2} \in V\left(\Lambda_{2}\right)$. There are vertices $\bar{u}_{1} \in V\left(\Lambda_{1}\right)$ and $\bar{u}_{2} \in V\left(\Lambda_{2}\right)$ such that $\iota_{1}\left(u_{1}\right) \in \operatorname{supp}\left(\psi\left(\bar{u}_{1}\right)\right)$ and $\iota_{2}\left(u_{2}\right) \in \operatorname{supp}\left(\psi\left(\bar{u}_{2}\right)\right)$. Since $\Lambda_{1}$ and $\Lambda_{2}$ are joined in $\Lambda_{1} * \Lambda_{2}, \psi\left(\bar{u}_{1}\right)$ and $\psi\left(\bar{u}_{2}\right)$ are commutative. Hence, $\operatorname{supp}\left(\bar{u}_{1}\right)$ and $\operatorname{supp}\left(\bar{u}_{2}\right)$ are contained in a single clique in $\Gamma$ by Lemma 2.2 . Thus, $\iota_{1}\left(u_{1}\right)$ and $\iota_{2}\left(u_{2}\right)$ are adjacent in $\Gamma$.

By $K_{n}$, we denote the complete graph on $n$ vertices. The right-angled Artin group on $K_{n}, A\left(K_{n}\right)$, is isomorphic to $\mathbb{Z}^{n}$.

Lemma 3.4. Let $\Gamma$ be a finite graph. Suppose that $\psi: A\left(K_{n}\right) \rightarrow A(\Gamma)$ is an embedding satisfying condition $(K K)$. Then there is a full embedding $\iota: K_{n} \rightarrow \Gamma$ with $\iota\left(K_{n}\right) \subset \operatorname{supp}(\psi)$.

Proof. Since $\psi$ satisfies condition (KK) and since $K_{n}$ is complete, $\operatorname{supp}(\psi)$ spans a clique on $l$ vertices of $\Gamma$ by Lemma 2.2 . Hence, we have an embedding $A\left(K_{n}\right) \cong$ $\mathbb{Z}^{n} \hookrightarrow \mathbb{Z}^{l} \hookrightarrow A(\Gamma)$. This implies $n \leq l$, and so we obtain an injective map $V\left(K_{n}\right) \rightarrow$ $\operatorname{supp}(\psi)$, which induces a full embedding $\iota: K_{n} \rightarrow \Gamma$ with $\iota\left(K_{n}\right) \subset \operatorname{supp}(\psi)$.

Proof of Proposition 3.2. We may assume that each of $\Lambda_{1}, \ldots \Lambda_{n}$ is a singleton graph and each of $\Lambda_{n+1}, \ldots, \Lambda_{m}$ has at least two vertices. Put $\Lambda_{0}:=\Lambda_{1} * \cdots * \Lambda_{n}$. Then $\Lambda_{0}$ is isomorphic to the complete graph on $n$ vertices, $K_{n}$. In addition, we can decompose $\Lambda$ into $\Lambda_{0} *\left(*_{i=n+1}^{m} \Lambda_{i}\right)$. Hence, we have $A(\Lambda)=A\left(\Lambda_{0}\right) \times A\left(\Lambda_{n+1}\right) \times$ $\cdots \times A\left(\Lambda_{m}\right)$. By restricting $\psi$ to the abelian factor $A\left(\Lambda_{0}\right)$, we obtain an embedding $\psi_{0}: A\left(\Lambda_{0}\right) \hookrightarrow A(\Gamma)$ satisfying condition (KK). Therefore, by Lemma 3.4 we obtain a full embedding $\iota_{0}: \Lambda_{0} \rightarrow \Gamma$ with $\iota_{0}\left(\Lambda_{0}\right) \subset \operatorname{supp}\left(\psi_{0}\right)$. Consider the family of full embeddings $\iota_{0}, \iota_{n+1}, \ldots, \iota_{m}$. Since each of $\Lambda_{n+1}, \ldots, \Lambda_{m}$ is irreducible and has at least two vertices, by repeatedly applying Lemma 3.3, we obtain the desired full embedding $\iota: \Lambda_{0} * \Lambda_{n+1} * \cdots * \Lambda_{m} \rightarrow \Gamma$.

\section{Proof of Theorem 1.3}

In this section we prove Theorem 1.3 . We first rephrase Theorem 1.3 in terms of join. Recall that $\left(\Lambda_{1} \sqcup \cdots \sqcup \Lambda_{m}\right)^{c}=\Lambda_{1}^{c} * \cdots * \Lambda_{m}^{c}$. Hence, if $\Lambda$ is the complement of a linear forest, namely, if $\Lambda$ is the complement of the disjoin union of finitely many path graphs, then $\Lambda$ is the join of the complements of finitely many path graphs. 


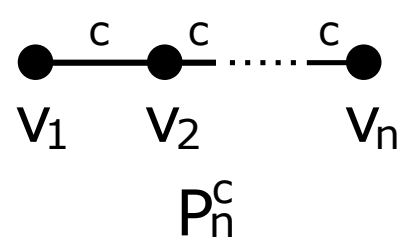

Figure 1. This picture illustrates $P_{n}^{c}$. Real lines with the characters $c$ represent the edges in $P_{n}=\left(P_{n}^{c}\right)^{c}$, each of which joins non-adjacent vertices in $P_{n}^{c}$. In this picture, any two distinct vertices not joined by a line are adjacent in $P_{n}^{c}$.

Theorem 4.1 (rephrased). Let $\Lambda$ be the join of the complements of finitely many path graphs and $\Gamma$ a finite graph. If $A(\Lambda) \hookrightarrow A(\Gamma)$, then $\Lambda \leq \Gamma^{e}$.

To obtain a full embedding $\Lambda \rightarrow \Gamma^{e}$ in the assertion above, we consider the join-component, the complement $P_{n}^{c}$ of the path graph $P_{n}$ on $n$ vertices.

Lemma 4.2. Let $n$ be a positive integer other than 3 and $\Gamma$ a finite graph. Suppose that $\psi: A\left(P_{n}^{c}\right) \rightarrow A(\Gamma)$ is an embedding satisfying condition (KK). Then there is a full embedding $\iota: P_{n}^{c} \rightarrow \Gamma$ with $\iota(v) \in \operatorname{supp}(\psi(v))\left(\forall v \in V\left(P_{n}^{c}\right)\right)$. In particular, $\iota\left(P_{n}^{c}\right) \subset \operatorname{supp}(\psi)$.

Proof. Let $\left\{v_{1}, v_{2}, \ldots, v_{n}\right\}$ be the vertices of $P_{n}^{c}$ labelled as illustrated in Figure 1.

The assertion is trivial in the case where $n=1$. Therefore, we may assume $n=2$ or $n \geq 4$. Suppose $n=2$. Then $P_{2}^{c}$ consists of two vertices $v_{1}, v_{2}$. If there is no full embedding $\iota: P_{2}^{c} \rightarrow \Gamma$ with $\iota\left(v_{1}\right) \in \operatorname{supp}\left(\psi\left(v_{1}\right)\right)$ and $\iota\left(v_{2}\right) \in \operatorname{supp}\left(\psi\left(v_{2}\right)\right)$, then $\operatorname{supp}\left(\psi\left(v_{1}\right)\right)$ and $\operatorname{supp}\left(\psi\left(v_{2}\right)\right)$ do not have distinct vertices $u_{1} \in \operatorname{supp}\left(\psi\left(v_{1}\right)\right)$ and $u_{2} \in \operatorname{supp}\left(\psi\left(v_{2}\right)\right)$ such that $\left\{u_{1}, u_{2}\right\} \notin E(\Gamma)$. Hence the $\operatorname{supports,} \operatorname{supp}\left(\psi\left(v_{1}\right)\right)$ and $\operatorname{supp}\left(\psi\left(v_{2}\right)\right)$, are contained in a single clique in $\Gamma$, and so $\psi\left(v_{1}\right)$ and $\psi\left(v_{2}\right)$ are commutative in $A(\Gamma)$. This implies that a non-trivial element $\left[v_{1}, v_{2}\right]:=v_{1} v_{2} v_{1}^{-1} v_{2}^{-1}$ of $A\left(P_{2}^{c}\right)=F_{2}$ is an element of the kernel of $\psi$, a contradiction.

We now assume $n \geq 4$. By $C_{i}$, we denote the clique in $\Gamma$ spanned by $\operatorname{supp}\left(\psi\left(v_{i}\right)\right)$. Since $\psi\left(v_{i}\right)$ and $\psi\left(v_{j}\right)$ are commutative when $|i-j|>1$, we obtain the following claim by Lemma 2.2 .

Claim 4.3. If $|i-j|>1$, then any vertex of $C_{i}$ and any vertex of $C_{j}$ are either identical or adjacent in $\Gamma$.

If $\Gamma$ has a sequence of mutually distinct vertices $y^{(1)}, y^{(2)}, \ldots, y^{(n)}$ such that $y^{(i)} \in$ $V\left(C_{i}\right)$ and that $y^{(i-1)}$ and $y^{(i)}$ are non-adjacent, then the map $\iota: P_{n}^{c} \rightarrow \Gamma$ defined by $\iota\left(v_{i}\right):=y^{(i)}(1 \leq i \leq n)$ determines an embedding $P_{n}^{c} \rightarrow \Gamma$ by Claim 4.3 . Since $y^{(i-1)}$ and $y^{(i)}$ are non-adjacent, $\iota: P_{n}^{c} \rightarrow \Gamma$ is a full embedding. Therefore we have only to prove that $\Gamma$ has a sequence of mutually distinct vertices $y^{(1)}, y^{(2)}, \ldots, y^{(n)}$ such that $y^{(i)} \in V\left(C_{i}\right)$ and that $y^{(i-1)}$ and $y^{(i)}$ are non-adjacent. 
Suppose, on the contrary, that

$(* *)$ the graph $\Gamma$ does not have a sequence of mutually distinct vertices $y^{(1)}, y^{(2)}, \ldots, y^{(n)}$ such that $y^{(i)} \in V\left(C_{i}\right)$ and that $y^{(i-1)}$ and $y^{(i)}$ are nonadjacent.

To deduce a contradiction, we will prove that the commutator $\left[\left(v_{1}\right)^{v_{2} v_{3} \cdots v_{n-1}}, v_{n}\right]$ is a non-trivial element of the kernel of $\psi$. We first observe that $\left[\left(v_{1}\right)^{v_{2} v_{3} \cdots v_{n-1}}, v_{n}\right]$ is non-trivial in $A\left(P_{n}^{c}\right)$. Since the preceding letter and succeeding letter of each letter $v^{\epsilon}$ in the word $\left[\left(v_{1}\right)^{v_{2} v_{3} \cdots v_{n-1}}, v_{n}\right]$ are not commutative with $v^{\epsilon}$, the word

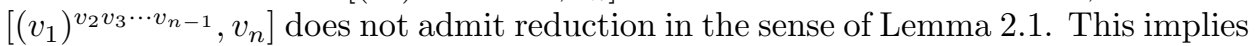
that the word $\left[\left(v_{1}\right)^{v_{2} v_{3} \cdots v_{n-1}}, v_{n}\right]$ is reduced and a non-trivial element in $A\left(P_{n}^{c}\right)$. Thus the remaining task is to show that $\left[\left(v_{1}\right)^{v_{2} v_{3} \cdots v_{n-1}}, v_{n}\right] \in \operatorname{ker} \psi$. To this end, it is enough to prove that the element $\psi\left(v_{1}\right)^{\psi\left(v_{2}\right) \psi\left(v_{3}\right) \cdots \psi\left(v_{n-1}\right)}$ can be represented as a word consisting of vertices of $C_{1}, \ldots, C_{n-1}$, each of which is commutative with all of the vertices of $C_{n}$. We first inductively define the family $Y^{(1)}, Y^{(2)}, \ldots, Y^{(n-1)}$ of (possibly empty) subsets of $V(\Gamma)$ as follows:

(i) $Y^{(1)}:=V\left(C_{1}\right)$.

(ii) Suppose that $Y^{(i-1)}$ is defined. Then we set

$$
Y^{(i)}:=\left\{y \in V\left(C_{i}\right) \mid \exists x_{y} \in Y^{(i-1)} \text { such that }\left\{x_{y}, y\right\} \notin E(\Gamma)\right\} .
$$

With regard to this family $Y^{(1)}, Y^{(2)}, \ldots, Y^{(n-1)}$, we claim that:

Claim 4.4. Any vertex of $C_{n}$ and any vertex in $\cup_{i=1}^{n-1} Y^{(i)}$ are either identical or adjacent in $\Gamma$.

Proof of Claim 4.4. Note that any vertex of $C_{n}$ and any vertex in $\cup_{i=1}^{n-2} Y^{(i)}$ are either identical or adjacent in $\Gamma$ by Claim 4.3 . So we have to show that if $Y^{(n-1)} \neq \emptyset$, any element $y^{(n-1)} \in Y^{(n-1)}$ is commutative with all vertices of $C_{n}$. By the construction of $Y^{(n-1)}$, there is an element $y^{(n-2)} \in Y^{(n-2)}$ which is not commutative with $y^{(n-1)}$. By repeating this argument, we can find a sequence $y^{(1)}, \ldots, y^{(n-1)}$ such that $y^{(i)} \in Y^{(i)}$, and that $y^{(i-1)}$ and $y^{(i)}$ are not commutative $(2 \leq i \leq n-1)$. Suppose, on the contrary, that there is an element $y^{(n)} \in V\left(C_{n}\right)$, which is not commutative with $y^{(n-1)}$. Then we can observe that $y^{(i)} \neq y^{(j)}$ if $i<j$ as follows. We first consider the case where $i=1$ and $2 \leq j \leq n-1$. Notice that the element $y^{(1)}$ is either identical with $y^{(j+1)}$ or adjacent to $y^{(j+1)}$ by Claim 4.3 . On the other hand, the element $y^{(j)}$ is non-adjacent to $y^{(j+1)}$. Hence, we obtain that $y^{(1)} \neq y^{(j)}$. We next consider the case where $i=1$ and $j=n$. Since $n \geq 4$, we have $n-1>2$. Although the element $y^{(n)}$ is non-adjacent to $y^{(n-1)}$, the element $y^{(1)}$ must be adjacent to $y^{(n-1)}$ by Claim 4.3 and the case where $i=1$ and $j=n-1$. Therefore $y^{(1)} \neq y^{(n)}$. In case $i \geq 2$, the element $y^{(i)}$ is non-adjacent to $y^{(i-1)}$, but the element $y^{(j)}$ is either adjacent to $y^{(i-1)}$ or identical with $y^{(i-1)}$. So $y^{(i)} \neq y^{(j)}$. Thus $y^{(i)} \neq y^{(j)}$ if $i<j$, and therefore the sequence $y^{(1)}, \ldots, y^{(n)}$ violates our assumption $(* *)$.

Fix reduced words $W_{i}$ in $V\left(C_{i}\right)$ representing $\psi\left(v_{i}\right)(1 \leq i \leq n)$. If two given words $w_{1}, w_{2}$ represent the same element in $A(\Gamma)$, then we denote by $w_{1}=w_{2}$. If $w_{1}, w_{2}$ are identical as words, then we denote by $w_{1} \equiv w_{2}$. We inductively construct words $\check{W}_{1}, \ldots, \check{W}_{n-1}$ satisfying the following conditions.

(W-1) $\breve{W}_{1}$ is a word in $Y^{(1)}$. Namely, the word $\check{W}_{1}$ consists of the vertices in $Y^{(1)}$. 
$(\mathrm{W}-i) \check{W}_{i}$ is a word in $Y^{(i)}$. Moreover,

$$
\check{W}_{i}^{-1} \cdots \check{W}_{2}^{-1} \check{W}_{1} \check{W}_{2} \cdots \check{W}_{i}=W_{i}^{-1} \cdots W_{2}^{-1} W_{1} W_{2} \cdots W_{i}
$$

as elements in $A(\Gamma)(i \geq 2)$.

Let us start the construction of the words $\check{W}_{1}, \ldots, \check{W}_{n-1}$.

(Step 1) $\check{W}_{1} \equiv W_{1}$. Obviously $\check{W}_{1}$ satisfies $(\mathrm{W}-1)$.

(Step 2) If $W_{2}$ is a word in $Y^{(2)}$, set $\check{W}_{2} \equiv W_{2}$. Then the word $\check{W}_{2}$ satisfies (W$2)$. We now suppose that $W_{2}$ is not a word in $Y^{(2)}$, i.e., there is a vertex $v \in V\left(C_{2}\right) \backslash Y^{(2)}$ such that the letter $v^{\epsilon}(\epsilon= \pm 1)$ is contained in $W_{2}$. We write $W_{2} \equiv w_{2} v^{\epsilon} w_{2}^{\prime}$. Then $W_{2}^{-1} \check{W}_{1} W_{2} \equiv\left(w_{2}^{\prime}\right)^{-1}\left(v^{\epsilon}\right)^{-1} w_{2} \check{W}_{1} w_{2} v^{\epsilon} w_{2}^{\prime}$. Note that the letter $v^{\epsilon}$ is commutative with $w_{2}$, because $C_{2}$ is a clique containing $v$. By the definition of $Y^{(2)}$, the letter $v^{\epsilon}$ is commutative with $\check{W}_{1}$. Hence, $W_{2}^{-1} \check{W}_{1} W_{2}=\left(w_{2}^{\prime}\right)^{-1}\left(w_{2}\right)^{-1} \check{W}_{1} w_{2} w_{2}^{\prime}$ in $A(\Gamma)$. If $w_{2} w_{2}^{\prime}$ is a word in $Y^{(2)}$, we set $\breve{W}_{2} \equiv w_{2} w_{2}^{\prime}$. If not, then applying the same reduction to $w_{2} w_{2}^{\prime}$ until we obtain a word $\check{W}_{2}$ in $Y^{(2)}$. Then $\check{W}_{2}$ satisfies the condition (W-2).

(Step $i$ ) Assume that $\check{W}_{1}, \ldots, \check{W}_{i-1}$ satisfy the conditions $(\mathrm{W}-1),(\mathrm{W}-2), \ldots,(\mathrm{W}-$ $(i-1))$, respectively. If $W_{i}$ is a word in $Y^{(i)}$, set $\check{W}_{i} \equiv W_{i}$. Then the word $\check{W}_{i}$ satisfies $(\mathrm{W}-i)$. We now suppose that $W_{i}$ is not a word in $Y^{(i)}$. Since $W_{i}$ is not a word in $Y^{(i)}$, there is a vertex $v \in V\left(C_{i}\right) \backslash Y^{(i)}$ such that the letter $v^{\epsilon}(\epsilon= \pm 1)$ is contained in $W_{i}$. So we write $W_{i} \equiv w_{i} v^{\epsilon} w_{i}^{\prime}$. Then we have the following equality:

$$
\begin{aligned}
& W_{i}^{-1} \check{W}_{i-1}^{-1} \cdots \check{W}_{2}^{-1} \check{W}_{1} \check{W}_{2} \cdots \check{W}_{i-1} W_{i} \\
\equiv & \left(w_{i}^{\prime}\right)^{-1}\left(v^{\epsilon}\right)^{-1}\left(w_{i}\right)^{-1} \check{W}_{i-1}^{-1} \cdots \check{W}_{2}^{-1} \check{W}_{1} \check{W}_{2} \cdots \check{W}_{i-1} w_{i} v^{\epsilon} w_{i}^{\prime} .
\end{aligned}
$$

Since $\check{W}_{1}, \ldots, \check{W}_{i-2}$ are words in $Y^{(1)}, \ldots, Y^{(i-2)}$, respectively, the letter $v^{\epsilon}$ is commutative with each of $\check{W}_{1}, \ldots, \check{W}_{i-2}$ by Claim 2.2 In addition, since $v \in V\left(C_{i}\right) \backslash Y^{(i)}$ and since $\check{W}_{i-1}$ is a word in $Y^{(i-1)}$, the letter $v^{\epsilon}$ is commutative with $\check{W}_{i-1}$. Furthermore, $v^{\epsilon}$ is commutative with $w_{i}$, because $C_{i}$ is a clique. Thus we have:

$$
\begin{aligned}
& \left(w_{i}^{\prime}\right)^{-1}\left(v^{\epsilon}\right)^{-1}\left(w_{i}\right)^{-1} \check{W}_{i-1}^{-1} \cdots \check{W}_{2}^{-1} \check{W}_{1} \check{W}_{2} \cdots \check{W}_{i-1} w_{i} v^{\epsilon} w_{i}^{\prime} \\
= & \left(w_{i}^{\prime}\right)^{-1}\left(w_{i}\right)^{-1} \check{W}_{i-1}^{-1} \cdots \check{W}_{2}^{-1} \check{W}_{1} \check{W}_{2} \cdots \check{W}_{i-1} w_{i} w_{i}^{\prime} .
\end{aligned}
$$

If $w_{i} w_{i}^{\prime}$ is a word in $Y^{(i)}$, we set $\check{W}_{i} \equiv w_{i} w_{i}^{\prime}$. If not, then applying the same reduction to $w_{i} w_{i}^{\prime}$ until we obtain a word $\check{W}_{i}$ in $Y^{(i)}$. In the end, $\check{W}_{i}$ obviously satisfies the condition $(\mathrm{W}-i)$.

By Claim 4.4 $\check{W}_{1}, \ldots, \check{W}_{n-1}$ are commutative with $W_{n}$, which is a representative of $\psi\left(v_{n}\right)$. Since $\psi\left(v_{1}\right)^{\psi\left(v_{2}\right) \psi\left(v_{3}\right) \cdots \psi\left(v_{n-1}\right)}$ is a multiplication of $\check{W}_{1}, \ldots, \check{W}_{n-1}$, it is

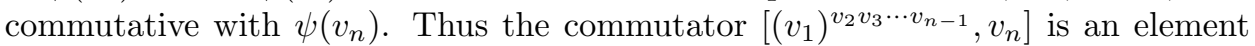
of $\operatorname{ker} \psi$, as desired.

To treat the case where $n=3$, we use the following lemma due to Kim-Koberda.

Lemma 4.5 ([4, Theorem 5.4]). Let $\Lambda$ be a finite graph whose right-angled Artin group $A(\Lambda)$ has no center. Suppose that $A(\Lambda)$ has an embedding into the direct product $G_{1} \times G_{2}$ of (non-trivial) groups $G_{1}, G_{2}$. If the natural projections $A(\Lambda) \rightarrow$ $G_{i}(i=1,2)$ have non-trivial kernels, then $\Lambda$ contains a full subgraph isomorphic to the cyclic graph of length 4 . 
Lemma 4.6. Let $\Gamma$ be a finite graph. Suppose that $\psi: A\left(P_{3}^{c}\right) \rightarrow A(\Gamma)$. Then there is a full embedding $\iota: P_{3}^{c} \rightarrow \Gamma$ with $\iota\left(P_{3}^{c}\right) \subset \operatorname{supp}(\psi)$.

Proof. For simplicity, we assume that $\operatorname{supp}(\psi)=V(\Gamma)$. Suppose, on the contrary, that

(A) $P_{3}^{c}$ is not a full subgraph of $\Gamma$. Namely, $P_{3}$ is not a full subgraph of $\Gamma^{c}$.

We first prove that $\Gamma^{c}$ is the disjoint union of finitely many complete graphs. Let $C$ be a connected component of $\Gamma^{c}$. If $\# V(C) \leq 2$, then the connectedness of $C$ obviously implies that $C$ is complete. So we may assume $\# V(C) \geq 3$. Pick two edges $e_{1}^{c}$ and $e_{2}^{c}$ of $C \leq \Gamma^{c}$ that share a vertex. Then, by our assumption (A), the set $e_{1}^{c} \cup e_{2}^{c}$ of vertices spans a triangle in $\Gamma^{c}$. In other words, the initial vertex and terminal vertex of any edge-path consisting of three vertices in $C$ is adjacent. By repeatedly using this fact, we can verify that, for any edge-path in $C$, the initial vertex is adjacent to the terminal vertex. Therefore the connected component $C$ must be complete. Thus, $\Gamma^{c}$ is the disjoint union of finitely many complete graphs, and so $\Gamma$ is the join of finitely many edgeless graphs. Hence, $A(\Gamma)$ is the direct product $A_{1} \times \cdots \times A_{m}$ of free groups $A_{1}, \ldots, A_{m}$. Since $A\left(P_{3}^{c}\right)$ is not free and since $A_{1} \times \cdots \times A_{m}$ contains an embedded $A\left(P_{3}^{c}\right)$, the integer $m$ is greater than 1. We now regard $A(\Gamma)$ as the direct product $\left(A_{1} \times \cdots \times A_{m-1}\right) \times A_{m}$ of two direct factors, $A_{1} \times \cdots \times A_{m-1}$ and $A_{m}$. Let $\pi_{m-1}, \pi_{m}$ denotes the projections $A\left(P_{3}^{c}\right) \rightarrow A_{1} \times \cdots \times A_{m-1}$ and $A\left(P_{3}^{c}\right) \rightarrow A_{m}$, respectively. Then since $A\left(P_{3}^{c}\right)$ is not free, the projection $\operatorname{ker} \pi_{m}$ must be non-trivial. Note that $A\left(P_{3}^{c}\right) \cong \mathbb{Z} * \mathbb{Z}^{2}$ has no center. If $\operatorname{ker} \pi_{m-1}$ is non-trivial, then by Lemma 4.5 . the defining graph $P_{3}^{c}$ must have a full subgraph isomorphic to the cyclic graph of length 4 , a contradiction. So we may assume that $\pi_{m-1}$ is injective. In other words, $A\left(P_{3}^{c}\right) \hookrightarrow A_{1} \times \cdots \times A_{m-1}$. Hence, by repeating this argument, we can reduce the number of the direct factors in the target group. Finally, we have that $A\left(P_{3}^{c}\right) \hookrightarrow A_{1}$, which is impossible.

Lemma 4.7. Let $\Lambda$ be the join of the complements of finitely many path graphs and $\Gamma$ a finite graph. Suppose that $\psi: A(\Lambda) \rightarrow A(\Gamma)$ is an embedding satisfying condition (KK). Then there is a full embedding $\iota: \Lambda \rightarrow \Gamma$ with $\iota(\Lambda) \subset \operatorname{supp}(\psi)$.

Proof. Suppose that $\Lambda_{1}, \ldots, \Lambda_{m}$ is the irreducible graphs such that $\Lambda=\Lambda_{1} * \cdots * \Lambda_{m}$ and $\Lambda_{i} \cong P_{n_{i}}^{c}(1 \leq i \leq m)$. Then by restricting $\psi$ to each direct factor $A\left(\Lambda_{i}\right)$, we obtain $\psi: A\left(\Lambda_{i}\right) \hookrightarrow A(\Gamma)$ with condition $(\mathrm{KK})$. Now by Lemma 4.2 and 4.6 , we obtain full embeddings $\iota_{i}: \Lambda_{i} \rightarrow \Gamma$ with $\iota_{i}\left(\Lambda_{i}\right) \subset \operatorname{supp}\left(\psi_{i}\right)$ for $(1 \leq i \leq m)$. Since $\Lambda_{1}^{c}, \ldots, \Lambda_{m}^{c}$ are path graphs, their connectedness together with Lemma 3.1 implies that $\Lambda_{1}, \ldots, \Lambda_{m}$ are irreducible. Thus, by applying Proposition 3.2 to $\Lambda=$ $\Lambda_{1} * \cdots * \Lambda_{m}$, we obtain the result that there is a full embedding $\iota: \Lambda \rightarrow \Gamma$ with $\iota(\Lambda) \subset \operatorname{supp}(\psi)$.

Proof of Theorem 1.3 (Theorem 4.1). Suppose that there is an embedding of the right-angled Artin group $A(\Lambda)$ on the join $\Lambda$ of the complements of finitely many path graphs into the right-angled Artin group $A(\Gamma)$ on a finite graph $\Gamma$. By Theorem 2.3 due to Kim-Koberda, we have an embedding $\psi: A(\Lambda) \hookrightarrow A\left(\Gamma^{e}\right)$ satisfying condition (KK), where $\Gamma^{e}$ is the extension graph of $\Gamma$. Consider the full subgraph $\Gamma^{\prime}$ of $\Gamma^{e}$, which is spanned by $\operatorname{supp}(\psi)=\cup_{v \in V(\Lambda)} \operatorname{supp}(\psi(v))$. Then we have an embedding $\psi: A(\Lambda) \hookrightarrow A\left(\Gamma^{\prime}\right)$ satisfying condition (KK). Now, by Lemma 4.7, we have $\Lambda \leq \Gamma^{\prime}$. Thus $\Lambda \leq \Gamma^{\prime} \leq \Gamma^{e}$, as desired. 


\section{REFERENCES}

[1] M. Casals-Ruiz, Embeddability and universal theory of partially commutative groups, Int. Math. Res. Not. IMRN 2015, no. 24, 13575-13622.

[2] J. Crisp and B. Wiest, Embeddings of graph braid and surface groups in right-angled Artin groups and braid groups, Algebr. Geom. Topol. 4 (2004), 439-472.

[3] T. Katayama, Right-angled Artin groups and full subgraphs of graphs, J. Knot Theory Ramifications, 26 (2017) 1750059, 22 pp.

[4] S. Kim and T. Koberda, Embedability between right-angled Artin groups, Geom. Topol. 17 (2013), no. 1, 493-530.

[5] E. Lee and S. Lee, Embeddability of right-angled Artin groups on complements of trees, preprint, available at arXiv: 1706.10002.

[6] H. Servatius. Automorphisms of graph groups, J. Algebra 126 (1989), no. 1, 34-60.

(Takuya Katayama) Department of Mathematics, Hiroshima University, 1-3-1 Kagamiyama, Higashi-Hiroshima, Hiroshima 739-8526, Japan

E-mail address: tkatayama@iroshima-u.ac.jp 